\title{
Advanced Pericardial Malignant Mesothelioma
}

National Cancer Institute

\section{Source}

National Cancer Institute. Advanced Pericardial Malignant Mesothelioma. NCI Thesaurus.

Code C8703.

A malignant mesothelioma extensively involving the pericardium. 